\title{
Probing the Fluctuations of Optical Properties in Time-Resolved Spectroscopy
}

\author{
Francesco Randi, ${ }^{1, \$}$ Martina Esposito, ${ }^{1}$ Francesca Giusti, ${ }^{1}$ Oleg Misochko, ${ }^{3,4}$ Fulvio Parmigiani, ${ }^{1,5,6}$ \\ Daniele Fausti, ${ }^{1,5, *}$ and Martin Eckstein ${ }^{2,7, \dagger}$ \\ ${ }^{1}$ Department of Physics, Università degli Studi di Trieste, 34127 Trieste, Italy \\ ${ }^{2}$ Max Planck Institute for the Structure and Dynamics of Matter, 22761 Hamburg, Germany \\ ${ }^{3}$ Institute of Solid State Physics, Russian Academy of Sciences, Chernogolovka, Moscow Region 142432, Russia \\ ${ }^{4}$ Moscow Institute of Physics and Technology (State University), Dolgoprudny, Moscow Region 141700, Russia \\ ${ }^{5}$ Sincrotrone Trieste SCpA, 34127 Basovizza, Italy \\ ${ }^{6}$ International Faculty, Universität zu Köln, 50937 Köln, Germany \\ ${ }^{7}$ Department of Physics, University of Erlangen-Nürnberg, 91058 Erlangen, Germany
}

(Received 23 May 2017; published 3 November 2017)

\begin{abstract}
We show that, in optical pump-probe experiments on bulk samples, the statistical distribution of the intensity of ultrashort light pulses after interaction with a nonequilibrium complex material can be used to measure the time-dependent noise of the current in the system. We illustrate the general arguments for a photoexcited Peierls material. The transient noise spectroscopy allows us to measure to what extent electronic degrees of freedom dynamically obey the fluctuation-dissipation theorem, and how well they thermalize during the coherent lattice vibrations. The proposed statistical measurement developed here provides a new general framework to retrieve dynamical information on the excited distributions in nonequilibrium experiments, which could be extended to other degrees of freedom of magnetic or vibrational origin.
\end{abstract}

DOI: 10.1103/PhysRevLett.119.187403

Pump-probe experiments are the primary way to study condensed matter out of its equilibrium state on time scales of femto- and picoseconds. In optical pump-probe experiments, ultrashort pulses are used in pairs. The pump triggers the dynamical response and the probe is used to detect changes in the optical properties of the sample. By and large, the experiments performed to date measure the intensity variation of probe pulses after their interaction with the sample for each pump-probe delay, following the protocol of averaging over many stroboscopically repeated experiments [1]. Little attention has been given to the accurate measurement of the fluctuation of the intensity of reflected (or transmitted) probe pulses.

In the basic implementation of a "statistical" pump-probe setup, the intensity of every single probe pulse is separately acquired with low-electronic-noise detectors, for every pump-probe delay. This allows us to measure both the average of the intensity, which gives the usual pump-probe signal [e.g., the relative variation of the reflectivity $\Delta R / R\left(t_{p}\right)$ as a function of pump-probe delay $\left.t_{p}\right]$, and its statistical distribution to all orders. Recent technological advances enabled experiments delivering such full statistical information on intensity fluctuations [2-5], thereby providing an experimental benchmark to address the following interesting, yet largely unexplored, question: What is the spectroscopic information carried by the fluctuations of the intensity of ultrashort light pulses reflected (or transmitted) by complex materials out of equilibrium?

It is known that the photon counting noise in the radiation emitted by nanoscopic emitters contains valuable information on their steady-state transport properties [6,7], but for pump-probe measurements in bulk samples it is still unclear how to connect the fluctuations of a measurement which is spatially averaged over the optical beam section to microscopic properties of the material. Proof-of-principle experiments in this direction have shown that, for transparent materials, i.e., materials where a large optical gap freezes the electronic degrees of freedom and an effective photon-phonon Raman coupling is the leading interaction, the statistical fluctuations of the probe intensity can be used to map the temporal evolution of vibrational observables $[2,3,8,9]$. Nevertheless, in the more general setting of pump-probe experiments on complex absorbing materials, where the photoexcitation can trigger a dynamical response in the electronic system, a formalism capable of linking the intensity fluctuations of the probe pulses to the microscopic properties of the material is still unavailable. In this Letter we provide such formalism and show how the knowledge of the fluctuations of the optical properties of a system can give access to the spectrum of current fluctuations, which is not accessible via the average intensity in a pump-probe experiment. The general theoretical result will be illustrated with numerical simulations and experimental data for photoinduced coherent lattice vibrations in a charge-density wave system.

Intensity fluctuations.-A pump-probe experiment measures the number $m$ of photocounts in a detector, during a detection time window that is very large compared to the probe-pulse duration (in general, larger by at least 3 orders of magnitude). In the statistical pump-probe experiment one obtains in addition the variance $(\Delta m)^{2}=\sigma[I]+\langle m\rangle$, which can be written as the sum of a shot-noise contribution 
$\langle m\rangle$ proportional to the intensity itself, and a sampledependent contribution $\sigma[I]$. Because the induced fields at the detector are linearly related to their sources, i.e., the current density $j$ in the sample, the probability distribution $\mathcal{P}(m)$ can be written in terms of the time-ordered current correlation functions [10]. In the following, we show that the nonequilibrium current fluctuations generated by the pump give rise to a contribution $\sigma_{\text {bulk }}$ to $\sigma[I]$ which can be identified by the statistical pump-probe experiment on a bulk sample, since it is of leading order in the sample volume. Because Maxwell's equations relate the current density $j(1)$ at one space-time point 1 to the induced fields $E_{\text {ind }}(2)=\int d 1 g(2,1) j(1)$ at a point 2 via a linear kernel $g(2,1)$, the result can be stated in the form $\left(1 \equiv\left(\boldsymbol{r}_{1}, t_{1}\right)\right.$, $\left.2 \equiv\left(\boldsymbol{r}_{2}, t_{2}\right), \ldots\right)$

$\sigma_{\text {bulk }}=\epsilon^{2} \int d t d t^{\prime} E_{+}(t) E_{-}\left(t^{\prime}\right)\left\langle\delta j(t, \boldsymbol{R}) \delta j\left(t^{\prime}, \boldsymbol{R}\right)\right\rangle_{\text {ret }}$,

where $\langle j(1) j(2) \cdots\rangle_{\text {ret }} \equiv \int d \overline{1} d \overline{2} g(1, \overline{1}) g(2, \overline{2})\langle j(\overline{1}) j(\overline{2}) \cdots\rangle$ is the expectation value of the currents propagated to spacetime points $1,2, \ldots, \boldsymbol{R}$ is the location of the detector, $\epsilon$ is proportional to the detection efficiency, and $E_{+}$is the positive frequency component $\sim e^{-i \omega t}$. To lowest order in the probe amplitude, the correlation function in Eq. (1) can be evaluated without the effect of the probe. Hence, Eq. (1) shows how the probe pulse can act as a filter to measure the transient current noise in the nonequilibrium state in a specific time window.

To prove Eq. (1) we start from Ref. [10], which gives the factorial moments $I_{1}=\langle m\rangle, I_{2}=\langle m(m-1)\rangle$ of the photocount distribution $\mathcal{P}$ in terms of the sources,

$$
\begin{aligned}
I_{n}= & \int d 1 d 1^{\prime} \cdots d n d n^{\prime} \mathcal{D}_{1,1^{\prime}} \cdots \mathcal{D}_{n, n^{\prime}} \\
& \times t\left\langle T_{\bar{\tau}}[j(1) \cdots j(n)] T_{\tau}\left[j\left(1^{\prime}\right) \cdots j\left(n^{\prime}\right)\right]\right\rangle_{\mathrm{ret}} .
\end{aligned}
$$

Here $T_{\tau}\left(T_{\bar{\tau}}\right)$ is the time-ordering (anti-time-ordering) operator acting on the sources, and $\mathcal{D}_{1,1^{\prime}}$ is the detector response function. For a small and wide-band detector at site $\boldsymbol{R}$, which absorbs at all positive frequencies, the latter is simply $\mathcal{D}_{1,1^{\prime}}=\epsilon \delta\left(\boldsymbol{r}_{1}-\boldsymbol{R}\right) \delta\left(\boldsymbol{r}_{1}{ }^{\prime}-\boldsymbol{R}\right) \int_{0}^{\infty} d \omega e^{-i \omega\left(t_{1}-t_{1}^{\prime}\right)}$. Differently from the standard quantum theory of photodetection [11,12], Eq. (2) is not obtained within the rotating wave approximation, and is thus applicable down to ultrashort (possibly single-cycle) pulses [10]. The variance of the photocount is $(\Delta m)^{2}=\left\langle m^{2}\right\rangle-\langle m\rangle^{2}=I_{2}-I_{1}^{2}+\langle m\rangle$, so that $\sigma[I]=I_{2}-I_{1}^{2}$. To evaluate $\sigma[I]$, we shift the operators $j(t)=\langle j(t)\rangle+\delta j(t)$, and expand $\sigma[I]$ in the fluctuations. In a bulk sample, terms like $\int d^{3} \boldsymbol{r}_{1} d^{3} \boldsymbol{r}_{2} \cdots\left\langle\delta j\left(\boldsymbol{r}_{1}\right) \delta j\left(\boldsymbol{r}_{2}\right) \cdots\right\rangle$ are proportional to the volume $V$, since they are obtained as the derivative of the extensive free energy with respect to an external vector potential. Hence, the dominant contribution $\left(\sim V^{3}\right)$ to $\sigma[I]$ is given by the second order terms like $\langle j(1)\rangle\langle\delta j(2) \delta j(3)\rangle\langle j(4)\rangle$ (first and zeroth order terms vanish by construction). Since $\langle j(\boldsymbol{R}, t)\rangle_{\text {ret }} \equiv E(t)$ is just the classical reflected field at the detector, and the contraction with the detector function selects the positive and negative frequency parts, $\int d 1^{\prime} \mathcal{D}_{1,1^{\prime}}\left\langle j\left(1^{\prime}\right)\right\rangle_{\text {ret }} \equiv E_{+}\left(t_{1}\right)$ and $\int d 1^{\prime} \mathcal{D}_{1^{\prime}, 1}\left\langle j\left(1^{\prime}\right)\right\rangle_{\text {ret }} \equiv E_{-}\left(t_{1}\right)$, one arrives at Eq. (1) [13] (see Supplemental Material [14]).

While Eq. (1) holds for an arbitrary geometry, to obtain specific results we adopt the standard setup and neglect propagation effects in the sample, so that $E_{\text {ind }}(t, \boldsymbol{R})=$ $-\dot{A}(t)$ with $A(t) \propto \int_{\text {sample }} d^{3} \boldsymbol{r} j\left(\boldsymbol{r}, t_{\text {ret }}\right)$, where $t_{\text {ret }}=t-\Delta t$ is shifted by the propagation time $\Delta t$ from the sample to the detector. This gives

$$
\sigma_{\text {bulk }} \propto \epsilon^{2} \int d t d t^{\prime}\left\langle\delta J\left(t_{\text {ret }}\right) \delta J\left(t_{\text {ret }}^{\prime}\right)\right\rangle \dot{E}_{+}(t) \dot{E}_{-}\left(t^{\prime}\right),
$$

where $J$ is the current integrated over the sample volume. Hence, $\sigma_{\text {bulk }}$ is determined by the current fluctuations $C\left(t, t^{\prime}\right)=\left\langle J\left(t^{\prime}\right) J(t)\right\rangle=\left\langle\delta J\left(t^{\prime}\right) \delta J(t)\right\rangle$, while the reflectivity is ultimately related, via the Kubo formula, to the timeresolved response function $\chi^{R}\left(t, t^{\prime}\right)=i \theta\left(t-t^{\prime}\right)\langle[J(t)$, $\left.\left.J\left(t^{\prime}\right)\right]\right\rangle$ [15]. To analyze $C\left(t, t^{\prime}\right)$ and $\chi^{R}\left(t, t^{\prime}\right)$, we introduce the Wigner transform $f\left(t_{p}, \omega\right)=(1 / 2 \pi) \int d s e^{i \omega s} f\left(t_{p}+s / 2\right.$, $\left.t_{p}-s / 2\right)$. In a thermal state, $C\left(t_{p}, \omega\right)$ and $\chi^{R}\left(t_{p}, \omega\right)$ are related by a fluctuation-dissipation theorem (FDT) [16],

$$
C\left(t_{p}, \omega\right)=2 b(\omega, \beta) \operatorname{Im} \chi^{R}\left(t_{p}, \omega\right),
$$

where $b(\omega, \beta)=\left(e^{\beta \omega}-1\right)^{-1}$ is the Bose function with inverse temperature $\beta$. A measurement with $\dot{E}_{ \pm}(t) \sim e^{\mp i \omega t}$ in Eq. (3) would then give $\sigma_{\text {bulk }} \propto C(\omega)$; i.e., $\sigma_{\text {bulk }}$ is negligible at room temperature for probe photon energies in the visible and infrared spectral range, and only the shot noise is relevant, as it is usually assumed in quantum optics for the reflection of light by a mirror. (Note that the sample can modify the shot noise, like an "imperfect reflector," but this corresponds to contributions to $\sigma[I]$ which are subleading in the volume compared to $\sigma_{\text {bulk }}$.) When the sample is out of equilibrium, instead, $\left\langle J(t) J\left(t^{\prime}\right)\right\rangle$ can significantly contribute to the total fluctuation of the intensity, and its measurement can therefore give information on the nonequilibrium state.

To illustrate such approach both from the theoretical and experimental point of view, we concentrate on a Peierls charge-density wave (CDW) system. A prototypical realization is bismuth [17], where an impulsive excitation generates coherent vibrations of the $A_{1 g}$ phonon, which modulates the out-of-equilibrium reflectivity [4]. A minimal theoretical model for the Peierls system is the Holstein model, which describes free electrons coupled to a dispersionless vibrational mode (Einstein phonon). At half filling, the Hamiltonian is 
$H=-t_{0} \sum_{\langle i j\rangle \sigma} c_{i \sigma}^{\dagger} c_{j \sigma}+\omega_{0} \sum_{j} b_{j}^{\dagger} b_{j}+g \sqrt{2} \sum_{j} X_{j}\left(n_{j}-1\right)$,

where $t_{0}$ is the nearest-neighbor hopping, $c_{j \sigma}^{\dagger}$ creates an electron at lattice site $j$ (spin $\sigma$ ), $b_{j}$ and $b_{j}^{\dagger}$ are the bosonic operators of a phonon mode at frequency $\omega_{0}$, and $g$ is the coupling constant between the electronic density $n_{j}=$ $n_{j \uparrow}+n_{j \downarrow}$ and the phonon coordinate $X_{j}=\left(b_{j}^{\dagger}+b_{j}\right)$. Below a critical temperature, the system is in an insulating and symmetry-broken phase, with a staggered charge disproportionation on neighboring sites and a gap at the Fermi level. We have numerically studied the outof-equilibrium dynamics of the Holstein model using the nonequilibrium dynamical mean-field theory [18] within the self-consistent Migdal approximation [19], on a bipartite lattice (see Supplemental Material [14]). We choose $g=0.34$, the inverse temperature $\beta=25$, and $\omega_{0}=0.2$. The free bandwidth $W=4$ sets the energy and time scale $(\hbar=1)$. The system is brought out of its equilibrium state by a short modulation of the hopping, $t_{0} \rightarrow t_{0}\left(1+\delta h e^{-(t-0.16)^{2} / 0.32}\right)$, see the dotted line in Fig 1(a). Since we focus only on the subsequent relaxation dynamics, the effect of this excitation is similar to experiments in which a laser pulse impulsively creates a conduction band electron population. In the present case, the excitation density is controlled by $\delta h$.

Before discussing the nonequilibrium current fluctuations, we verify that this model captures the main features which are known from experiments on CDW systems [4],


FIG. 1. (a) Atomic displacement $\langle X\rangle$ as a function of time $t$, for different excitation strength $\delta h$ above and below the critical excitation $\delta h_{c}=1.7$ for melting of the CDW. The dotted line shows the hopping modulation (in arbitrary units) used to impulsively stimulate the system. (b) Oscillation frequency $\omega^{*}$ as a function of $\delta h$. The grey vertical line indicates $\delta h_{c}$, where a critical slow down is observed. i.e., a coherent oscillation which is increasingly damped and softened for larger excitation density. A good observable to study this dynamics is the displacement $\langle X\rangle$ of the atoms from the translationally invariant positions of the high-temperature phase $(\langle X\rangle$ takes opposite values on the two sublattices of the bipartite lattice). As shown in Fig. 1(a), for $\delta h$ below a critical value $\delta h_{c} \simeq 1.7,\langle X(t)\rangle$ coherently oscillates around a new nonzero position. ( $\delta h_{c} \simeq 1.7$ corresponds to a pump-induced conduction band population $n_{e x}=0.05$ ). As the excitation density increases, the oscillations are damped more quickly, and the frequency $\omega^{*}$ becomes lower; i.e. the vibrational mode is softened [see Fig. 1(b)]. For $\delta h>\delta h_{c}$, the melting of the CDW phase is induced, with oscillations around the undistorted atomic position $\langle X\rangle=0$ [Fig. 1(a)]. While the melting of the CDW phase has been observed in experiment [20], we will report measurements below the threshold and therefore focus on the regime $\delta h<\delta h_{c}$ for the following theoretical analysis.

In addition to the distortion, we calculate the optical susceptibility $\chi^{R}\left(t_{p}, \omega\right)$ and the fluctuations $C\left(t_{p}, \omega\right)$, as defined above Eq. (4). At equilibrium, the optical susceptibility $\chi^{R}(\omega)$ of the CDW phase displays a gapped optical band [Fig. 2(a)]. After the excitation, the out-of-equilibrium $\operatorname{Im} \chi\left(t_{p}, \omega\right)$ oscillates between a gapped spectrum, which is partially filled by intraband transitions due to the excited conduction band electron population [Fig. 2(a), $t_{p}=76$ ], and an almost gapless spectrum when the atoms are closest to their translationally invariant positions, $\langle X\rangle=0$ [Fig. 2(a), $\left.t_{p}=52\right]$. The spectrum $C\left(t_{p}, \omega\right)$ shows that current fluctuations exist only at low frequencies (bold black line) in equilibrium, but extend to high frequencies when a nonthermal population is created.

It is nontrivial to understand on which time scale correlated electrons can be considered to be in an effectively thermal state after an excitation, in particular in systems with strongly coupled electrons and phonons, where the phonons themselves are far from equilibrium. Theoretically, a check of the FDT (4) provides a natural way to access the thermal nature of a nonequilibrium state $[18,21]$. The dashed lines in Fig. 2(b) show the right-hand side of Eq. (4) for two representative pump-probe delays $\left(t_{p}=52\right.$ and 76), with a best-fit inverse temperature $\beta_{f}=$ 7.1 and 6.3, respectively. The most pronounced violation of the fluctuation dissipation relation is visible after one full oscillation (yellow curve), i.e., when $\langle X(t)\rangle$ is maximum [see also the blue line in the inset of Fig. 2(b), which shows a fit error between $C\left(t_{p}, \omega\right)$ and the right-hand side of Eq. (4)]. One can understand this behavior as follows: Initially, a nonthermal population is created at the top of the lower band and at the bottom of the upper band of the system. As the atoms move closest to $\langle X\rangle=0$ and the gap is the smallest, electrons thermalize and the occupation of the bands closely resembles the Fermi-Dirac distribution, with a high electronic temperature (see Supplemental 



FIG. 2. Optical susceptibility $\chi^{R}\left(t_{p}, \omega\right)$ (a) and current fluctuations (b) at equilibrium (black), and for different $t_{p}(\delta h=1.5)$. Dashed lines in (b) correspond to the right-hand side of the FDT (4), with $\beta_{f}=7.1$ and 6.3 for $t_{p}=52$ and 76 , respectively. Inset: Violation of the FDT (blue line, see text), $\langle X(t)\rangle$ (dashed line), and reflectivity fluctuations $\sigma_{\text {bulk }}[$ Eq. (3)] at probe frequency $\omega=1.3$ as a function of $t_{p}$.

Material [14] for additional details). However, when the gap revives after one period, a nonthermal distribution far from the Fermi energy is restored, because reopening of the gap itself prevents electronic thermalization along with the evolution of $\langle X\rangle$.

The measurement of the fluctuations of the intensity of the probe pulses now allows us to obtain the fluctuations of the current in the sample experimentally. In the present case, the nonthermal nature of the state after one oscillation period can be detected by probing the fluctuations at a specific high frequency. We evaluate Eq. (3) assuming a generic pulse $\dot{E}(t) \propto \sin \left[\omega_{p}\left(t-t_{p}\right)\right] e^{-\left(t-t_{p}\right)^{2} / \delta t^{2}}$ with frequency $\omega_{p}=1.3$ larger than the gap, probe time $t_{p}$, and probe pulse duration $\delta t=2.4$ [22]. The result, shown by the black curve in the inset of Fig. 2(b), closely resembles the error in the fit of the FDT. These numerical predictions can be compared to experimental results obtained in experiments on $\mathrm{Bi}$ single crystals with the statistical pump-probe setup [2] (see Supplemental Material [14]). The system is excited and probed at a photon energy of $1.5 \mathrm{eV}$, with a probe duration of 80 fs. Figures 3(a) and 3(b) show the measured variance $\Delta m$ and $\Delta R / R$ for two different pump fluences. The pump generates a coherent amplitude mode oscillation, which is seen in $\Delta R / R \propto\langle X\rangle . \Delta m$ also mainly follows this oscillation. This is expected because, in addition to the

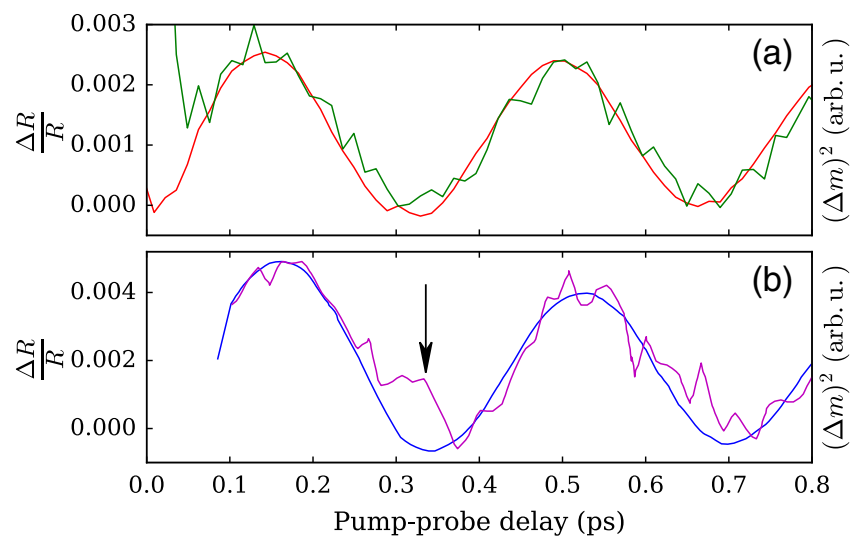

FIG. 3. Measured reflectivity change $\Delta R / R$ and variance $(\Delta m)^{2}$ in a bismuth crystal after optical excitation at a lower (a) and higher (b) excitation intensity. The curves $(\Delta m)^{2}$ are rescaled to obtain a best fit to $\Delta R / R$. The arrow points at enhanced fluctuations around the first oscillation period.

contribution $\sigma_{\text {bulk }}$ from the nonequilibrium current fluctuations, $\Delta m$ contains shot noise proportional to the intensity itself. Moreover, there is a slight variation with $\Delta R / R$, because the measurement of $\Delta m$ is calibrated to the equilibrium reflectivity of the sample. These contributions to $\Delta m$ are quantitatively unknown, but they are proportional to $\Delta R / R$, and one can clearly see that a rescaled $\Delta R / R$ cannot describe the time-dependent fluctuations $\Delta m$. In particular, we find an enhancement of the fluctuations at early times and, at higher fluence, after one oscillation period, i.e., around the time when the atoms revive to their most distorted positions. This is well in agreement with the theoretical prediction for the fluctuation contribution $\sigma_{\text {bulk }}$ which comes from the current noise. The experimental data therefore show that electrons have not thermalized at each time during the early CDW oscillations of the system. Longlived nonthermal electrons in Bi may be related to local conduction band minima at various points in the Brillouin zone [23]. Angular-resolved photoemission spectroscopy can provide a direct probe of electron distributions [24], but it is restricted to certain momenta, making the fluctuation measurement an important complementary tool to probe the nonequilibrium state.

In conclusion, in this Letter we have derived an expression for the fluctuations of the intensity of light reflected by a sample in an ultrafast pump-probe experiment. We have shown that fluctuations of the reflectivity in addition to shotnoise contributions can measure the nonequilibrium current noise spectrum as a function of time and frequency on the femtosecond time scale. In a straightforward way, current fluctuations can reveal the nonequilibrium state of the sample, such as the effective temperature of the relevant degrees of freedom, or whether the system can be considered to be in a quasithermal state at all. We illustrated our general results for the case of coherent vibrations in solids, both via numerical calculations and statistical pump-probe experiments on bismuth single crystals. We find current fluctuations at high 
energies, which reveals that nonthermalized electronic distributions emerge as the CDW gap oscillates. An intriguing future direction would be to also characterize the quantum state of the low-energy mode. While the theoretical simulations predict that it does not only coherently oscillate but also becomes squeezed, this is only indirectly reflected in the probed response at high energies. In general, the results of this Letter show that measuring the fluctuations in the reflectivity opens up an independent way to characterize the nonequilibrium dynamics in bulk solids, with intriguing future applications in the characterization of transient superconducting or nonthermal symmetry broken states.

F. R. conducted the theoretical research at the Max Planck Institute for the Structure and Dynamics of Matter (Hamburg) and the experimental research at the University of Trieste and the Elettra Synchrontron (Trieste). We thank Federico Cilento for the support in the setup of the experiments and useful discussions. Further, we thank Arunangshu Debnath, Fabio Benatti, Roberto Floreanini, Kelvin Titimbo, and Klaus Zimmerman for the insightful discussion. F. R. was supported by the Friuli Venezia Giulia Region (European Social Fund, operative program 2007/ 2013). D. F. aknowledges the support by the European Commission through the ERCStG2015, INCEPT, Grant No. 677488. M.E. acknowledges support by the DFG within the Sonderforschungsbereich 925 (project B4).

*Corresponding author.

daniele.fausti@elettra.eu

† Corresponding author.

martin.eckstein@fau.de

${ }^{\ddagger}$ Present address: Department of Physics, Princeton University, 08540 Princeton, New Jersey, USA.

[1] C. Giannetti, M. Capone, D. Fausti, M. Fabrizio, F. Parmigiani, and D. Mihailovic, Ultrafast optical spectroscopy of strongly correlated materials and high-temperature superconductors: A non-equilibrium approach, Adv. Phys. 65, 58 (2016).

[2] M. Esposito, K. Titimbo, K. Zimmermann, F. Giusti, F. Randi, D. Boschetto, F. Parmigiani, R. Floreanini, F. Benatti, and D. Fausti, Photon number statistics uncover the fluctuations in non-equilibrium lattice dynamics, Nat. Commun. 6, 10249 (2015).

[3] F. Randi, Master's thesis, Università degli Studi di Trieste, 2013.

[4] O. V. Misochko, Coherent phonons and their properties, J. Exp. Theor. Phys. 92, 246 (2001).

[5] C. Riek, P. Sulzer, M. Seeger, A. S. Moskalenko, G. Burkard, D. V. Seletskiy, and A. Leitenstorfer, Subcycle quantum electrodynamics, Nature (London) 541, 376 (2017).

[6] C. W. J. Beenakker and H. Schomerus, Counting Statistics of Photons Produced by Electronic Shot Noise, Phys. Rev. Lett. 86, 700 (2001).

[7] A. V. Lebedev, G. B. Lesovik, and G. Blatter, Statistics of radiation emitted from a quantum point contact, Phys. Rev. B 81, 155421 (2010).
[8] F. Benatti, M. Esposito, D. Fausti, R. Floreanini, K. Titimbo, and K. Zimmermann, Generation and detection of squeezed phonons in lattice dynamics by ultrafast optical excitations, New J. Phys. 19, 023032 (2017).

[9] K. Titimbo, Ph.D. thesis, Università degli Studi di Trieste, 2015, http://hdl.handle.net/10077/10941.

[10] M. Fleischhauer, Quantum-theory of photodetection without the rotating wave approximation, J. Phys. A 31, 453 (1998).

[11] P. L. Kelley and W. H. Kleiner, Theory of electromagnetic field measurement and photoelectron counting, Phys. Rev. 136, A316 (1964).

[12] L. Mandel, Fluctuations of photon beams and their correlations, Proc. Phys. Soc. London 72, 1037 (1958).

[13] Counterrotating terms $\sim E_{+} E_{+}$have been omitted, as they vanish in a typical pump-probe experiment which averages over the carrier envelope phase of the probe $E_{+} \propto e^{i \phi}$.

[14] See Supplemental Material at http://link.aps.org/ supplemental/10.1103/PhysRevLett.119.187403 for additional details about the derivation of equations, the numerical calculations, and the experimental setup.

[15] M. Eckstein and M. Kollar, Theory of time-resolved optical spectroscopy on correlated electron systems, Phys. Rev. B 78, 205119 (2008).

[16] R. Kubo, Statistical-mechanical theory of irreversible processes. I. General theory and simple applications to magnetic and conduction problems, J. Phys. Soc. Jpn. 12, 570 (1957).

[17] R. E. Peierls, More Surprises in Theoretical Physics (Princeton University Press, Princeton, NJ, 1991).

[18] H. Aoki, N. Tsuji, M. Eckstein, M. Kollar, T. Oka, and P. Werner, Nonequilibrium dynamical mean-field theory and its applications, Rev. Mod. Phys. 86, 779 (2014).

[19] Y. Murakami, P. Werner, N. Tsuji, and H. Aoki, Interaction quench in the Holstein model: Thermalization crossover fdrom electron- to phonon-dominated relaxation, Phys. Rev. B 91, 045128 (2015).

[20] S. W. Teitelbaum, T. Shin, J. Wolfson, Y. H. Cheng, I. J. Porter, M. Kandyla, and K. A. Nelson, A photoinduced symmetric crystalline phase in bismuth, arXiv:1609.04048.

[21] J. Kogoj, L. Vidmar, M. Mierzejewski, S. A. Trugman, and J. Bonča, Thermalization after photoexcitation from the perspective of optical spectroscopy, Phys. Rev. B 94, 014304 (2016).

[22] Note that because $\delta t$ is considerably smaller than one oscillation period of the phonon $\left(\sim 100 \mathrm{~J}^{-1}\right)$ but longer than the period $2 \pi / \omega_{p}$ of the probe pulse (i.e., the frequency uncertainty is small compared to $\left.\omega_{p}\right)$, the result approximately corresponds to a measurement of $C\left(t_{p}, \omega_{p}\right)$ itself, independent of the precise pulse shape.

[23] I. Timrov, T. Kampfrath, J. Faure, N. Vast, C. R. Ast, C. Frischkorn, M. Wolf, P. Gava, and L. Perfetti, Thermalization of photoexcited carriers in bismuth investigated by time-resolved terahertz spectroscopy and $a b$ initio calculations, Phys. Rev. B 85, 155139 (2012).

[24] J. Faure, J. Mauchain, E. Papalazarou, M. Marsi, D. Boschetto, I. Timrov, N. Vast, Y. Ohtsubo, B. Arnaud, and L. Perfetti, Direct observation of electron thermalization and electron-phonon coupling in photoexcited bismuth, Phys. Rev. B 88, 075120 (2013). 